\title{
A Prototype Sensor for In Situ Sensing of Fine Particulate Matter and Volatile Organic Compounds
}

\author{
Chee-Loon $\mathrm{Ng}^{1, *}$, Fuu-Ming Kai ${ }^{2}$, Ming-Hui Tee ${ }^{1}$, Nicholas Tan ${ }^{1}$ and Harold F. Hemond ${ }^{3}$ \\ 1 Singapore-MIT Alliance for Research and Technology (SMART) Centre, 1 CREATE Way, CREATE Tower, \\ \#10-01, Singapore 138602, Singapore; minghui1991@hotmail.com (M.-H.T.); \\ nicholastanyijian@hotmail.com (N.T.) \\ 2 Agency for Science, Technology and Research (A*STAR), National Metrology Centre (NMC), \\ 1 Science Park Drive, Singapore 118221, Singapore; kai_fuu_ming@nmc.a-star.edu.sg \\ 3 Parsons Laboratory, Massachusetts Institute of Technology, Room 48-425, 15 Vassar Street, Cambridge, \\ MA 02139, USA; hfhemond@exchange.mit.edu \\ * Correspondence: kelvinng@smart.mit.edu; Tel.: +65-6516-5230
}

Received: 16 December 2017; Accepted: 16 January 2018; Published: 18 January 2018

\begin{abstract}
Air pollution exposure causes seven million deaths per year, according to the World Health Organization. Possessing knowledge of air quality and sources of air pollution is crucial for managing air pollution and providing early warning so that a swift counteractive response can be carried out. An optical prototype sensor (AtmOptic) capable of scattering and absorbance measurements has been developed to target in situ sensing of fine particulate matter (PM2.5) and volatile organic compounds (VOCs). For particulate matter testing, a test chamber was constructed and the emission of PM2.5 from incense burning inside the chamber was measured using the AtmOptic. The weight of PM2.5 particles was collected and measured with a filter to determine their concentration and the sensor signal-to-concentration correlation. The results of the AtmOptic were also compared and found to trend well with the Dylos DC 1100 Pro air quality monitor. The absorbance spectrum of VOCs emitted from various laboratory chemicals and household products as well as a two chemical mixtures were recorded. The quantification was demonstrated, using toluene as an example, by calibrating the AtmOptic with compressed gas standards containing VOCs at different concentrations. The results demonstrated the sensor capabilities in measuring PM2.5 and volatile organic compounds.
\end{abstract}

Keywords: volatile organic compounds sensor; fine particulate matter sensor; in situ real-time air quality sensor; optical sensor; multi-pass absorbance; scattering

\section{Introduction}

Air pollution is a major environmental problem causing over seven million premature deaths every year [1]. The main air pollutants include criteria pollutants (i.e., particulate matter, carbon monoxide, ozone, nitrogen dioxide, sulfur dioxide, and lead) listed in the National Ambient Air Quality Standards [2] and other hazardous air pollutants (e.g., benzene, toluene, ethyl benzene, formaldehyde, naphthalene, acetaldehyde, trichloroethylene, and tetrachloroethylene) emitted from outdoor sources (e.g., industrial facilities, refineries, gas stations, vehicles, and wildfires) and indoor sources (e.g., building, construction materials, upholstered furniture and wood products, and fuel combustion for cooking or heating) [2-4]. To protect public health and to control emission sources, criteria pollutants and some of the hazardous air pollutants are usually monitored by conventional networks of static and sparse air quality monitoring stations [2]. Advancement towards smaller and portable sensing technologies at lower cost with capabilities to detect critical pollutants could greatly improve both temporal and spatial air pollution monitoring networks [5-9]. 
Common gas sensing technologies include optical methods, gas chromatography, and electrochemical approaches (e.g., metal-oxide semiconductors, polymers, carbon nanotubes) [5,10]. The optical method is one of the promising approaches because of its high accuracy, strong sensitivity, low environmental interference (e.g., temperature, humidity), and longer lifetime $[6,7,10]$. In this work, we developed a novel optical sensing system (AtmOptic) to detect fine particulate matter and some common hazardous gases (e.g., toluene) utilizing optical scattering and the multipass absorbance principle of sensing, respectively. Scattering is a physical process whereby some forms of radiation (e.g., light) are forced to deviate from a straight trajectory by one or more paths due to localized non-uniformities (e.g., particulate matter) in the medium through which they pass. The multipass absorbance method is used to measure weak spectra in gases or liquids by multiplying the effective path length through a medium and thereby increase absorbance.

\section{Materials and Methods}

\subsection{Instrument}

The layout of the AtmOptic is shown in Figure 1. The optical functions of the AtmOptic rely on the combination of two custom-designed flow cells, a scattering cell fitted with a light-emitting diode (LED) of $780 \mathrm{~nm}$ wavelength for particulate matter (PM) measurements using nephelometry and a broadband ( 185 to $1100 \mathrm{~nm}$ ) light source that illuminates a multipass cell constructed with two concave mirrors for volatile organic compound (VOC) measurements. Flow into the cells is via an inlet fine particle-sorting cyclone and a pathway that contains a rectangular bend to minimize the entrance of stray light. The flow passed through the scattering cell, multipass cell, and a particle collector before being released back to the atmosphere. Light from the scattering cell is observed with an amplified photodetector (Thorlabs, Newton, NJ, USA, Model PDF10A/M) and the data are recorded with an in-house microcontroller made with an Arduino UNO running custom software (pAtmOptic). Light from the multipass cell is observed with a spectrometer (Ocean Optics, Dunedin, FL, USA, USB4000) and the data are recorded with a single-board computer manufactured by Technologic systems (Fountain Hills, AZ, USA, Model TS-7260-64-128F) running custom software (iLEDLIF) developed by $[11,12]$. For continuous monitoring, a dual AIRPO (sgbotics, Singapore, Model S2028B) vacuum pumps draws air samples into the cells manifold. Particles are collected with Millipore ${ }^{\circledR}$ glass-fiber filters (Merck Millipore, Sigma-Aldrich, Singapore, Type 5, Lot 3110) and the humidity is measured with a temperature-corrected humidity sensor (ANSAC, Singapore, Model 104100-00).

\section{(a) AtmOptic}

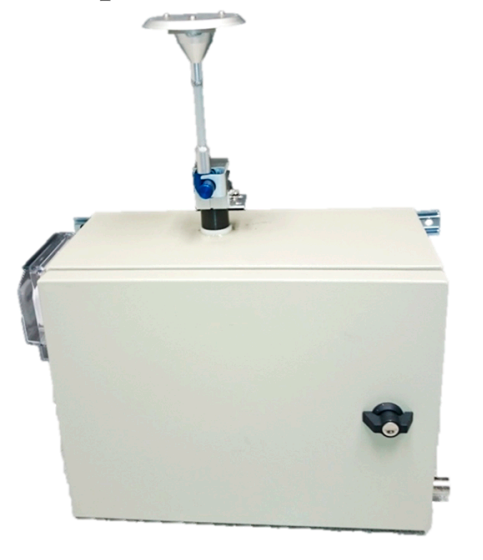

(b) Block Diagram

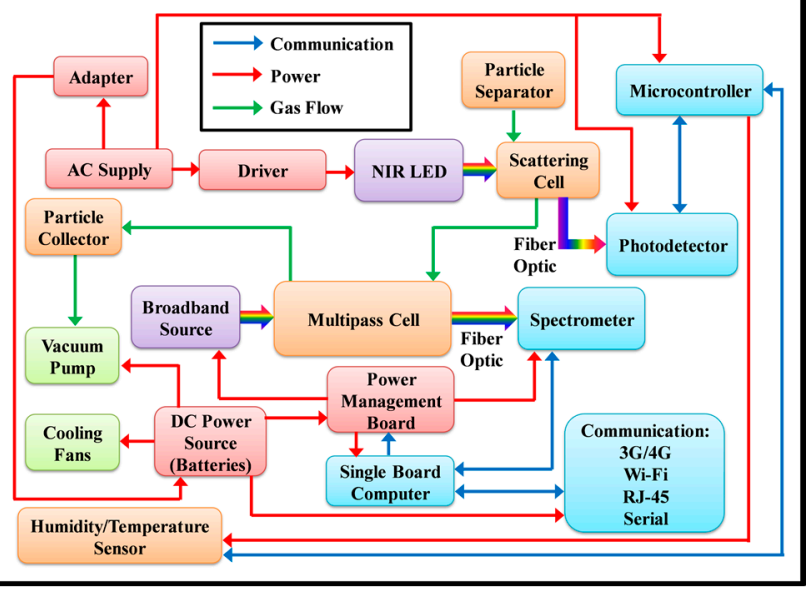

Figure 1. (a) Front views of the AtmOptic packaged inside a $40 \times 15 \times 30 \mathrm{~cm}$ enclosure for fixed location sensing; (b) Block diagram of the AtmOptic. 


\subsection{Fine Particulate Matter}

The experimental setup of the AtmOptic scattering response to fine particulate matter (PM2.5) concentration is shown in Figure 2. PM2.5 refers to particulate matter of $2.5 \mu \mathrm{m}$ or less in diameter. A $2.35 \times 0.63 \times 0.82 \mathrm{~m}$ in-house test chamber constructed with plexiglas reinforced with an aluminum frame was used to calibrate the AtmOptic for PM2.5 sensing. The AtmOptic continuously drew an air sample from an outlet centered at the base of the chamber. The flow rate is measured with a Key Instruments Polycarbonate flowmeter (RS, Singapore, Model MR3A14BVBN). Ambient air entered the chamber through an inlet centered at the lid of the chamber. An incense stick (旺里香) purchased from a local store in Singapore was burned at the four corners (insert graph in Figure 2), and the emissions produced were mixed by an Ebmpapst fan (element 14, Singapore, Model 4650X) instrumented below the inlet of the chamber to promote the mixing of smoke particles to achieve homogeneity. Each sampling cycle consists of a 30 min background air monitoring period $(t<0)$, the combustion period $(0 \leq t \leq T)$, and a 60 min post-burning period $(t \geq T)$; following the experimental procedure of [13]. The averaged concentration (i.e., mass per volumetric of air) of PM2.5 covering the combustion and post-burning period of each run was computed by measuring the mass of the Millipore ${ }^{\circledR}$ glass-fiber filter with a Mettler Toledo XP6 (Cole-Parmer, Vernon Hills, IL, USA) microbalance before and after the mass collection for each run and the volumetric flow rate through the AtmOptic.

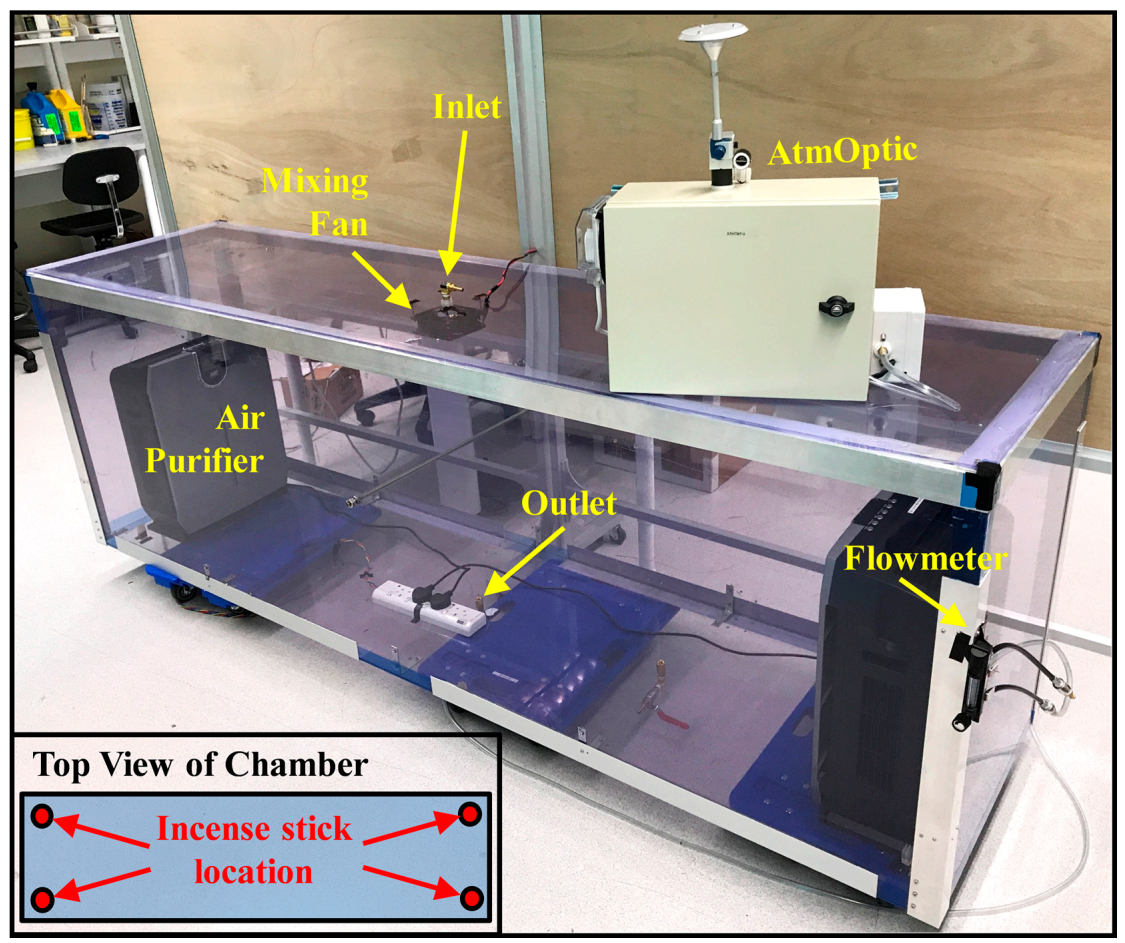

Figure 2. Experimental setup to study AtmOptic scattering response to PM2.5 concentration produced by burning incense sticks. Insert schematic shows the PM sources (incense sticks) locations.

The chamber was thoroughly cleaned with damp disposable paper towel after each run. Two American Air Filter (AAF) air purifiers (AAF Singapore, Singapore, Model PurAir 400A) equipped with six layers of High Efficiency Particulate Air (HEPA) filtration technology capable of covering a total area of 80 to $120 \mathrm{~m}^{3}$ were used to remove air particles inside the chamber for $25 \mathrm{~min}$ after each run. The background air in the chamber was checked with the AtmOptic after the cleaning and the percent difference in the baseline signals were found to never exceed $1.7 \%$. For all experiments, the background air signals in the chamber were monitored for $30 \mathrm{~min}$ and used for baseline correction and the flow rate was maintained at $1.5 \mathrm{~L} / \mathrm{min}$ throughout the experiment with a regulated control. 
For instrument comparison, the PM2.5 air sample measured by the AtmOptic was passed into the Dylos air quality monitor (Qtech Integrated, Singapore, Model DC1100 Pro) for particle counting before it was collected with the glass-fiber filter for mass measurement. It is not feasible to quantify the exact properties of each microscopic particle being counted by the Dylos. Therefore, it was assumed that (1) all particles are spherical with a density of $1.65 \times 10^{12} \mu \mathrm{g} / \mathrm{m}^{3}$ [14]; (2) the radius of the particle in the PM2.5 channel is $0.44 \mu \mathrm{m}$ [15]; and (3) $0.01 \mathrm{ft}^{3}$ is converted to $\mathrm{m}^{3}$ by multiplying the factor 3531.5 . The mass of a PM2.5 particle can then be computed as $5.89 \times 10^{-7} \mu \mathrm{g}$ and the PM2.5 concentration $\left(C_{P M 2.5}\right)$ can be obtained using:

$$
C_{P M 2.5}=3531.5(\text { No. of Particles })\left(5.89 \times 10^{-7}\right)
$$

Dylos was chosen for comparison because it was found to compare reasonably well with the air management system (AMS) in Philadelphia [16].

\subsection{Volatile Organic Compounds}

All experiments were performed using ultra high purity air (Leeden National Oxygen Ltd., Singapore) as the reference gas. The VOC vapor produced by the aqueous solution or solid state source was continuously drawn into the AtmOptic by the vacuum pump of the sensor when it was placed near the sensor inlet. For cargenogenic VOC (e.g., Benzene), the experiment was performed inside a fumehood (VEC Environment Pte Ltd., Singapore, Model Dynaflow GRP). For VOC (i.e., toluene in synthetic air) and ultra-high-purity air in compressed gas tanks, the gas was transferred from the tank to the inlet of the AtmOptic via tubing. After testing, the gas from the AtmOptic was exhausted to the fumehood through tubing. The AtmOptic was purged with ultra-high-purity air after each run to ensure the signal returned to the baseline before the next experiment.

\subsubsection{Laboratory Chemicals}

Eight VOC spectra (vapor phase) were recorded using the AtmOptic: (1) Acetone; (2) Benzene; (3) Methanol; (4) Naphthalene; (5) O-xylene; (6) Phenol; (7) Styrene; and (8) Toluene. Acetone (CAS 67-64-1, Item No. A1084-1-4000) was obtained from Orëc (Duga Products and Services Co., Ban Suan, Chongburi, Thailand), benzene (CAS 71-43-2, Lot 1219765525, Prod. 100515F) was from VWR International (Singapore), methanol (CAS 67-56-1, Product No. 179337, Lot SHBH4599V) was from Sigma-Aldrich (Singapore), naphthalene (CAS 91-20-3, Lot A0266833) was from Acros Organics (Fisher Scientific, Singapore), O-xylene (CAS 95-47-6, Lot 1413783) was from Sigma-Aldrich (Singapore), phenol (CAS 108-95-2, Lot 114Y009) was from Alfa-Aesar (Fisher Scientific, Singapore), styrene (CAS 100-42-5, Lot STBF-4819V) was from Sigma-Aldrich (Singapore), and toluene (CAS 108-88-3, Lot SHBF-4825V) from Sigma-Aldrich (Singapore). Compressed gas tanks containing different concentrations of toluene in synthetic air (Leeden National Oxygen Ltd., Singapore) from the BTEX (benzene, toluene, ethylbenzene, and $O$-xylene) group were used to demonstrate the quantification of VOC with the AtmOptic and the same calibration procedure can be repeated for other chemicals of interest.

\subsubsection{Household Products}

The VOC spectra of various household products were recorded using the AtmOptic: (1) Elmer's extra strong spray adhesive (Product No. E455); (2) 7CF interior/exterior spray paint (Color 39 black, Product No. R-8088); (3) Innisfree eco nail remover; (4) Araldite epoxy (5 min rapid cure); (5) Brasso metal polish; and (6) Gas lighter fluid. The test was performed by pouring an arbitrary amount of the products into a separate container and placing it near to the inlet of the AtmOptic for measurement. 


\section{Results and Discussion}

\subsection{Fine Particulate Matter}

Figure 3a shows that the background signal at the baseline was stable when there is no incense burning and the PM2.5 (if any) in the ambient was constant in the absence of the incense smoke. During the combustion period $(0 \leq t \leq T)$, the PM2.5 in the tank started to increase and the scattering signal rose to the maximum at the end of the combustion period. Post-burning, the signal began to drop over time as particle decay was observed. Figure $3 b$ shows that the peak maximum of the PM2.5 signal increases with the amount of burned incense. The end of the burning time varies within $126 \mathrm{~s}$ (blue shaded region) for all sampling cases. Once the incense sticks were lit in the chamber, the PM2.5 concentration increased rapidly until the last incense stick burned out at $t=T$. The averaged concentration of PM2.5 corresponded to the time covering both the combustion and post-burning periods. Figure $3 c$ shows that the integral signal is a linear function of the averaged concentration. There was no apparent adverse effect on the two measurements obtained without fan mixing. Figure $3 \mathrm{~d}$ shows that the concentration per millivolt $\left[C_{\text {per } m V}\right.$, in $\left.\mu \mathrm{g} /\left(\mathrm{m}^{3} \cdot \mathrm{mV}\right)\right]$ of the AtmOptic decreases linearly with the increase in averaged concentration $\left(C_{\text {Total }}\right)$ when it is less than $7933 \mu \mathrm{g} / \mathrm{m}^{3}$ :

Uncorrected:

$$
C_{\text {per mV }}=-1.3476 \times 10^{-4} C_{\text {Avg }}+2.9432
$$

Thereafter, the concentration per millivolt remained constant at $C_{\text {per } m V}=1.8872 \mu \mathrm{g} /\left(\mathrm{m}^{3} \cdot \mathrm{mV}\right)$.

Figure 3e compares a PM2.5 measurement of the AtmOptic with the Dylos. The total mass of PM2.5 collected by the filter was $1.05 \mathrm{mg}$ over a volume of $0.1234 \mathrm{~m}^{3}$ computed based on a constant flow rate of $1.5 \mathrm{~L} / \mathrm{min}$, resulting in an averaged concentration of $8511 \mu \mathrm{g} / \mathrm{m}^{3}$. Both instruments appeared to capture a similar emission profile with a small variation in concentration at a given time. Unlike AtmOptic, the Dylos observed a constant concentration closed to $100 \mu \mathrm{g} / \mathrm{m}^{3}$ post-burning and particle decay was not observed by the instrument. By applying the trapezoidal rule $\left[\left(t_{2}-t_{1}\right)\left(\frac{C_{2}-C_{1}}{2}\right)\right.$; where $t_{1}$ is previous time step, $t_{2}$ is current time step, $C_{1}$ is previous concentration value, and $C_{2}$ is current concentration value] to approximate the area under the curve and using the observed constant flow rate of $1.5 \mathrm{~L} / \mathrm{min}$, the total mass of PM2.5 computed with the data of both instruments were $0.88 \mathrm{mg}$, a $16.2 \%$ lower than the that $(1.05 \mathrm{mg})$ collected by the filter. Relative humidity (RH) could be a factor causing the discrepancy because the experiments were conducted around a $\mathrm{RH}$ between $50 \%$ and $70 \%$. Day et al. found that aerosol light scattering measurements were a function of $\mathrm{RH}$, particularly for RH values above 60\% [17].

\subsection{Volatile Organic Compounds}

Figure 4a shows the absorbance of different VOC peaks at different wavelengths, demonstrating that it is possible to detect and classify different VOCs using the multipass absorbance method in the AtmOptic. Figure $4 \mathrm{~b}$ compares the absorbance peak of various VOCs with published values and found them to be in very good agreement. Figure $4 \mathrm{c}$ shows that the absorbance of toluene in a toluene and acetone gas mixture peaks at $262 \mathrm{~nm}$, a red shift of $3 \mathrm{~nm}$ relative to a pure toluene gas, which may be associated with the function of admixture concentrations. The acetone absorbance in the mixture peaks at $280 \mathrm{~nm}$ and remained unchanged relative to a pure acetone gas. Exposure to this gas mixture may be encountered in a chemical manufacturer facility that produces a ready-to-use acetone-toluene solvent mixture. Figure $4 \mathrm{~d}$ shows the absorbance spectra of toluene in a synthetic air peak between 259 and $261 \mathrm{~nm}$. Figure 4e shows that the absorbance peak obeys the Beer-Lambert [18] equation [ $A=\varepsilon l C$, where $A$ is absorbance (absorbance unit), $\varepsilon$ represents absorptiviity, $l$ denoted optical path length, and $C$ is concentration] up to $100 \mathrm{ppm}$. Each data point in the graph is an average of five measurements. Figure $4 \mathrm{f}$ shows that the absorbance spectra of different VOCs' presence in various household products peaks at different wavelengths, demonstrating the potential specificity of the AtmOptic in detecting these products. Both the Elmer's glue and Innisfree eco nail remover contain 
acetone showing an absorbance peak at 278 and $280 \mathrm{~nm}$, respectively. The 7CF interior/exterior spray paint contain toluene that peaks at 219 and $259 \mathrm{~nm}$. The Araldite epoxy peaks at $252 \mathrm{~nm}$. The Brasso metal polish peaks at 218 and $262 \mathrm{~nm}$. The gas lighter fluid contains butane and peaks at $251 \mathrm{~nm}$.

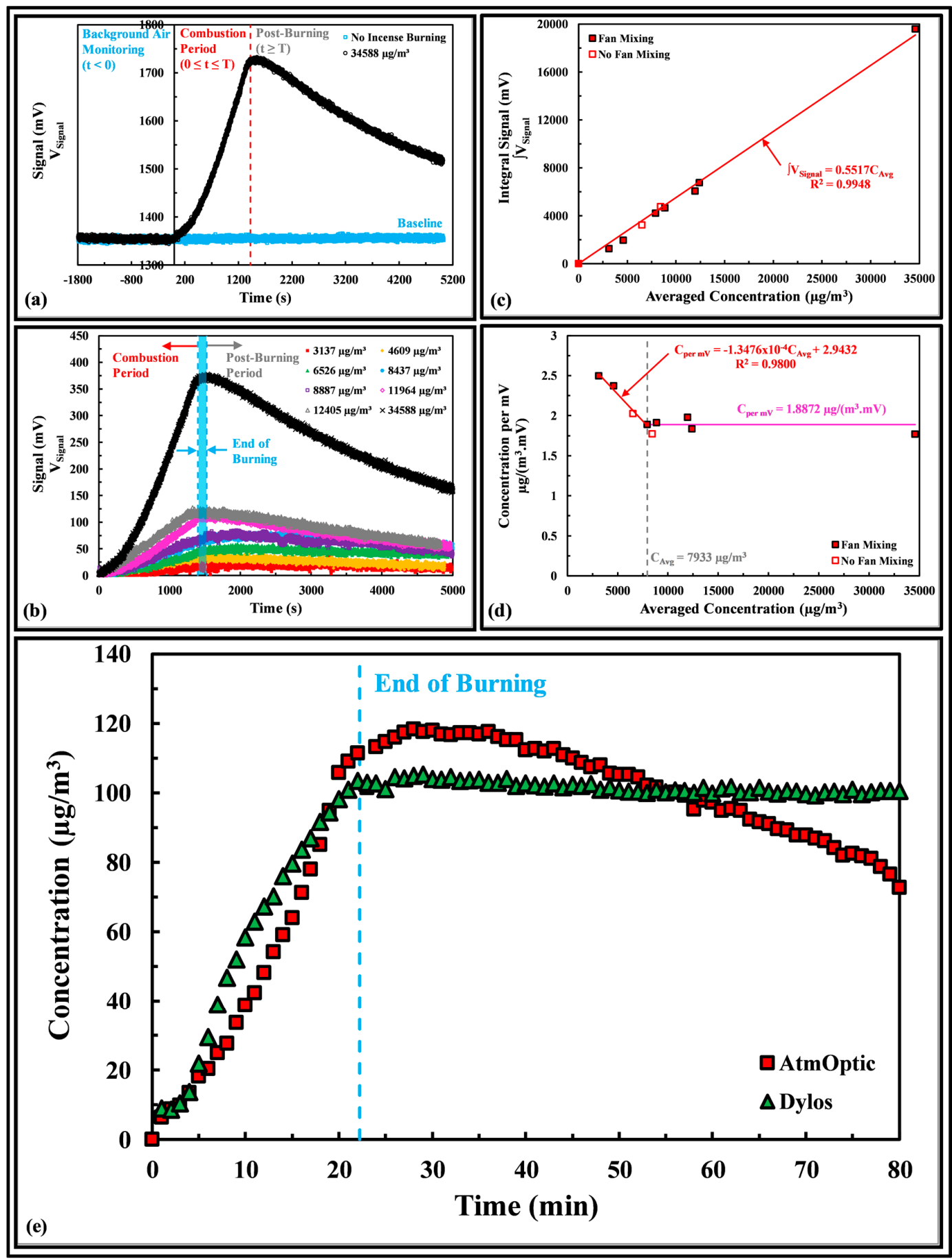

Figure 3. (a) Background air qualification and measured signal of seven incense sticks burning as a function of time; (b) Measured signal of the averaged concentration of PM2.5 produced by burning various numbers of incense sticks as a function of time. Legend: averaged concentration of PM2.5; (c) Integral signal as a function of averaged concentration of PM2.5, with and without fan mixing; (d) Concentration per millivolt as a function of the averaged concentration of PM2.5, with and without fan mixing; (e) Instruments comparison for PM2.5 measurements at an averaged concentration of $8511 \mu \mathrm{g} / \mathrm{m}^{3}$. 

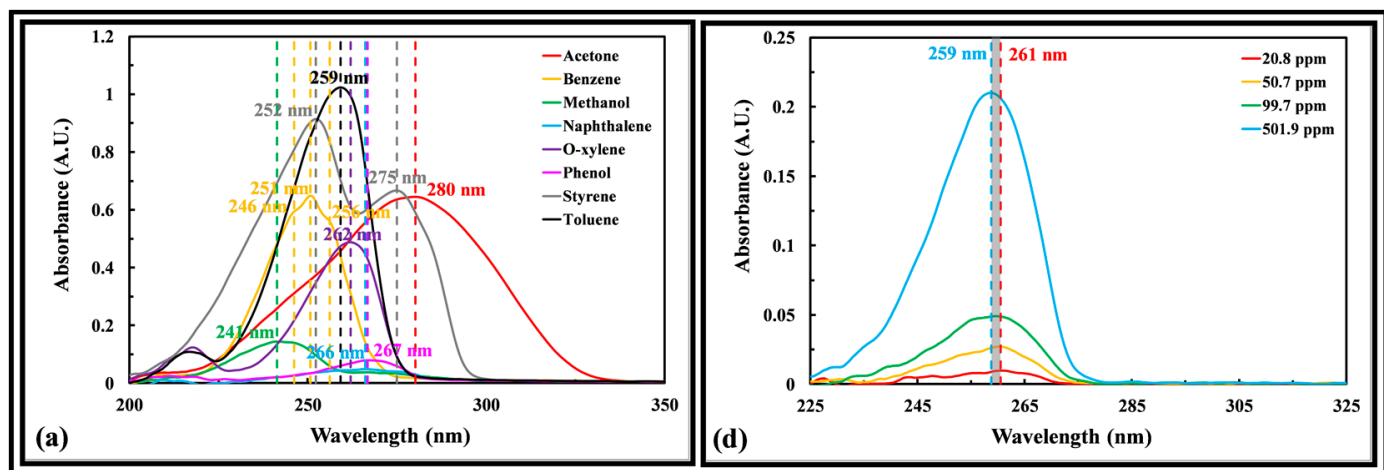

\begin{tabular}{|c|c|c|c|c|}
\hline \multirow{2}{*}{ VOCs } & \multicolumn{2}{|c|}{ Absorbance Peak (nm) } & \multirow{2}{*}{$\begin{array}{c}\% \\
\text { Difference }\end{array}$} & \multirow{2}{*}{ Reference } \\
\hline & Atm0ptic & Reference Value & & \\
\hline Acetone & 280 & 274 & 2.2 & [19] \\
\hline Benzene & $\begin{array}{c}246,251, \\
256\end{array}$ & $-, 255,-$ & 1.6 & [19] \\
\hline Methanol & 241 & - & - & - \\
\hline Naphthalene & 209,266 & -268 & 0.79 & [20] \\
\hline O-xylene & 218,262 & -264 & 0.64 & [21] \\
\hline Phenol & 211,267 & -270 & 1.2 & [19] \\
\hline Styrene & 252,275 & $245,-$ & $2.9,-$ & [22] \\
\hline b) Toluene & 217,259 &,- 261 & -0.71 & [23] \\
\hline
\end{tabular}
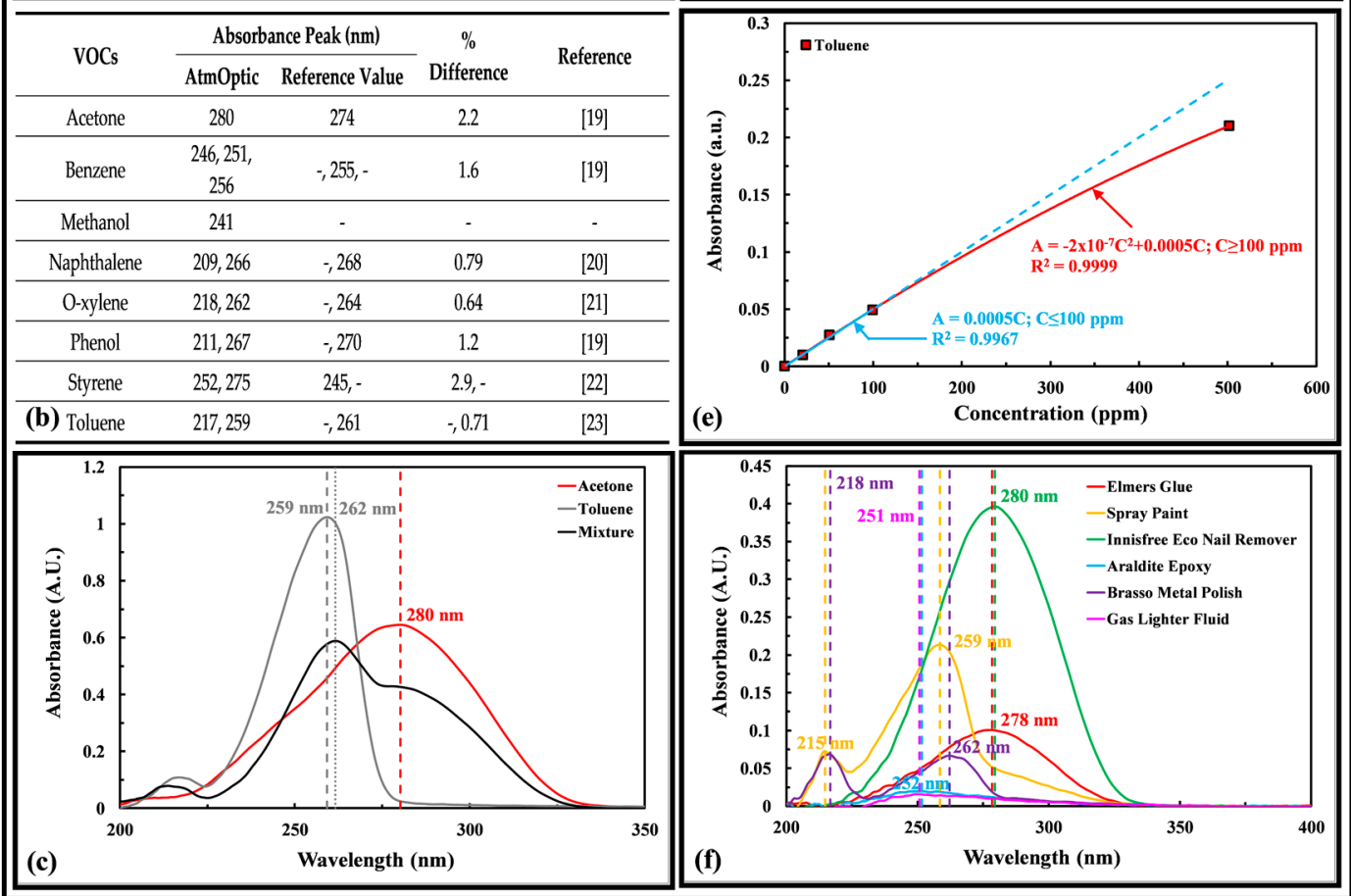

Figure 4. (a) Absorbance spectra of various VOCs measured with the AtmOptic; (b) Comparison of the AtmOptic absorbance peak with various references [19-23]; (c) Absorbance spectra of an acetone and toluene mixture; (d) Absorbance spectra of toluene of different concentrations; (e) Toluene absorbance peaks as a function of concentration; (f) Absorbance spectra of various household products.

\section{Conclusions}

The results described illustrate the AtmOptic's capabilities in measuring both the PM2.5 and VOCs present in the air, and suggest that it is possible to identify and quantify fine particulate matter and various VOCs using the optical method. When fully characterized, the AtmOptic can be used for sensing transboundary haze, VOC discharge from industry, and VOCs in consumer products and gas stations, among others. Matrix effects and changes in environmental conditions in these measurements were not investigated in this paper, which could be important and subsequently studied. For larger particles (e.g., PM10), scattering increases in the forward direction and decreases in the backward (light-facing) direction due to constructive and destructive interference. The exact relationship can be established through calibration using similar procedure as for PM2.5 and the replacement of a PM10 cyclone in the AtmOptic. Finally, a spectrum separation algorithm can be applied to improve the quantification of VOCs. 
Acknowledgments: Funding for this work and the costs of publication in open access were provided by the National Research Foundation Singapore under its Campus for Research Excellence and Technological Enterprise programme through the Singapore-MIT Alliance for Research and Technology's Innovation Centre and Center for Environmental Sensing and Modeling Interdisciplinary research group.

Author Contributions: In this work, Chee-Loon Ng and Fuu Ming Kai conceived the idea; Chee-Loon Ng designed and constructed the sensor, designed the experiments, performed data analysis and interpretation, and drafted the manuscript; Fuu Ming Kai provided knowledge on air quality measurement techniques and suggestions on experimentation; Ming Hui Tee developed the data acquisition board for the particulate matter detector; Nicholas Tan constructed the test chamber and performed the experiments; Harold F. Hemond provided the lab facilities and feedback to the manuscript.

Conflicts of Interest: The authors declare no conflict of interest.

\section{References}

1. World Health Organization (WHO). New Release: World Health Assembly Closes, Passing Resolutions on Air Pollution and Epilepsy. 2015. Available online: http:/ /www.who.int/mediacentre/news/releases / 2015/wha-26-may-2015/en/ (assessed on 15 August 2017).

2. U.S. Environmental Protection Agency (USEPA). National-Scale Air Toxics Assessment, and Summary of Results for the 2011 National-Scale Assessment. 2015. Available online: http:/ / www.epa.gov/national-airtoxics-assessment/2011-national-air-toxics-assessment (assessed on 15 August 2017).

3. World Health Organization (WHO). WHO Guidelines for Indoor Air Quality: Selected Pollutants; World Health Organization Regional Office for Europe: Copenhagen, Denmark, 2010.

4. Williams, R.; Kilaru, V.; Snyder, E.; Kaufman, A.; Dye, T.; Rutter, A.; Russell, A.; Hafner, H. Air Sensor Guidebook; EPA/600/R-14/159; U.S. Environmental Protection Agency: Washington, DC, USA, 2014.

5. Aleixandre, M.; Gerboles, M. Review of small commercial sensors for indicative monitoring of ambient gas. Chem. Eng. Trans. 2012, 30, 169-174.

6. MacDonell, M.; Raymond, M.; Wyker, D.; Finster, M.; Chang, Y.-S.; Raymond, T.; Temple, B.; Scofield, M. Mobile Sensors and Applications for Air Pollutants; EPA/600/R-14/051; U.S.; Environmental Protection Agency: Washington, DC, USA, 2013.

7. Snyder, E.; Watkins, T.H.; Solomon, P.A.; Thoma, E.D.; Williams, R.; Hagler, G.S.W.; Shelow, D.; Hindin, D.A.; Kilaru, V.J.; Peter, W. The changing paradigm of air pollution monitoring. Environ. Sci. Technol. 2013, 47, 11369-11377. [CrossRef] [PubMed]

8. Kumar, P.; Morawska, L.; Martani, C.; Biskos, G.; Neophytou, M.; Sabatino, S.D.; Bell, M.; Norford, L.; Britter, R. The rise of low-cost sensing for managing air pollution in cities. Environ. Int. 2015, 75, 199-205. [CrossRef] [PubMed]

9. Williams, R.; Kaufman, A.; Garvey, S. Next Generation Air Monitor (NGAM) VOC Sensor Evaluation Report; EPA/600/R-15/122; U.S. Environmental Protection Agency: Washington, DC, USA, 2015.

10. Liu, W.; Cheng, S.; Liu, H.; Hu, S.; Zhang, D.; Ning, H. A survey on gas sensing technology. Sensors 2012, 12, 9635-9665. [CrossRef] [PubMed]

11. Ng, C.L.; Hemond, H.F.; Senft-Grupp, S. Highly Compact Multi-Optical-Junction Optical Flowcell and Flexibly Deployable Optical Sensing Assemblies and Systems for In-Situ Real-Time Spectroscopic Measurements. Patent Corporation Treaty Application PCT/SG2012/000142, 20 April 2012.

12. Ng, C.L.; Senft-Grupp, S.; Hemond, H.F. A multi-platform optical sensor for in situ sensing of water chemistry. Limnol. Oceanogr. Methods 2012, 10, 978-990. [CrossRef]

13. See, S.W.; Balasubramanian, R. Characterization of fine particle emissions from incense burning. Build. Environ. 2011, 46, 1074-1080. [CrossRef]

14. Tittarelli, A.; Borgini, A.; Bertoldi, M.; De Saeger, E.; Ruprecht, A.; Stefanoni, R.; Tagliabue, G.; Contiero, P.; Crosignani, P. Estimation of Particle Mass Concentration in Ambient Air Using a Particle Counter. Atmos. Environ. 2008, 42, 8543-8548. [CrossRef]

15. Lee, J.Y.; Shin, H.J.; Bae, S.Y.; Kim, Y.P.; Kang, C.-H. Seasonal Variations of Particle Size Distributions of PAHs at Seoul, South Korea. Air Qual. Atmos. Health 2008, 1, 57-68. [CrossRef]

16. Air Quality Sensor Network for Philadelphia-Data Validation. Available online: http://www.fijnstofmeter. com/documentatie/Data-Validation.pdf (accessed on 13 September 2017). 
17. Day, D.E.; Malm, W.C.; Kreidenwais, S.M. Aerosol Light Scattering Measurements as a Function of Relative Humidity. J. Air Waste Manag. Assoc. 2000, 50, 710-716. [CrossRef] [PubMed]

18. Lakowicz, J.R. Principles of Fluorescence Spectroscopy, 3rd ed.; Springer: Berlin, Germany, 2006.

19. Kshitij Education India. Effect of Conjucation on $\lambda$ max. Available online: http://www.kshitij-iitjee.com/ effect-of-conjugation (accessed on 15 August 2017).

20. NIST Chemistry Webbook, SRD 69. Naphthalene. Available online: http://webbook.nist.gov/cgi/cbook. cgi? Name=naphthalene\&Units=SI\&cTG=on\&cUV=on (accessed on 15 August 2017).

21. Camou, S.; Tamechika, E.; Horiuchi, T. Portable Sensor for Determining Benzene Concentration from Airborne Liquid Samples with High Accuracy. NTT Tech. Rev. 2012, 10. Available online: https:/ / www.nttreview.jp/archive/ntttechnical.php?contents=ntr201202fa7.html (accessed on 15 August 2017).

22. NIST Chemistry Webbook, SRB69. Styrene. Available online: http://webbook.nist.gov/cgi/cbook.cgi? Name=styrene\&Units=SI\&cTG=on\&cUV=on (accessed on 15 August 2017).

23. NIST Chemistry Webbook, SRD 69. Toluene. Available online: http://webbook.nist.gov/cgi/cbook.cgi? Name $=$ toluene\&Units $=S I \& c T G=$ on\&cUV $=$ on (accessed on 15 August 2017).

(C) 2018 by the authors. Licensee MDPI, Basel, Switzerland. This article is an open access article distributed under the terms and conditions of the Creative Commons Attribution (CC BY) license (http://creativecommons.org/licenses/by/4.0/). 